Ger J Exerc Sport Res $2020 \cdot 50: 477-479$ https://doi.org/10.1007/s12662-020-00681-8 Eingegangen: 25 . September 2020 Angenommen: 30 . September 2020 Online publiziert: 16 . Oktober 2020 (c) Der/die Autor(en) 2020

Wilhelm Bloch ${ }^{1}$ - Anja Hirschmüller ${ }^{2}$ Frank Mayer ${ }^{3}$ - Andreas Nieß ${ }^{4} \cdot$ Tim Meyer $^{5}$. Claus Reinsberger ${ }^{6} \cdot$ Kai Röcker $^{7} \cdot$ Jürgen M. Steinacker ${ }^{8}$

${ }^{1}$ Institut für Kreislaufforschung und Sportmedizin, Deutsche Sporthochschule Köln, Köln, Deutschland

${ }^{2}$ Klinik für Orthopädie und Unfallchirurgie, Universitätsklinikum Freiburg, Freiburg im Breisgau, Deutschland

${ }^{3}$ Hochschulambulanz, Zentrum für Sportmedizin, Universität Potsdam, Potsdam, Deutschland

${ }^{4}$ Medizinische Klinik, Abteilung Sportmedizin, Universitätsklinikum Tübingen, Tübingen, Deutschland

${ }^{5}$ Institut für Sport- und Präventivmedizin, Universität des Saarlands, Saarbrücken, Deutschland

${ }^{6}$ Department Sport \& Gesundheit, Universität Paderborn, Paderborn, Deutschland

${ }^{7}$ Institut für Angewandte Gesundheitsförderung und Bewegungsmedizin, Hochschule Furtwangen, Furtwangen, Deutschland

${ }^{8}$ Sektion Sport- und Rehabilitationsmedizin, Universitätsklinikum Ulm, Ulm/Donau, Deutschland

\title{
Leserbrief zu Thieme, L. Jung stirbt, wen die Götter lieben? Ger J Exerc Sport Res 50, 280-296 (2020). https://doi.org/10.1007/s12662- 020-00654-x
}

\section{Leserbrief zu}

Thieme, L (2020) Jung stirbt, wen die Götter lieben? Ger J Exerc Sport Res 50, 280-296. https://doi.org/10.1007/s12662-020-00654-x

\section{Leserbrief des Wissen- schaftsrats der Deutschen Gesellschaft für Sportmedizin und Prävention (DGSP)}

Der o.g. Artikel berührt wichtige wissenschaftliche Positionen des Fachs Sportmedizin. Aus diesem Grund möchte der Wissenschaftsrat der DGSP (WR) im folgenden Text dazu Stellung nehmen.

Der WR bedankt sich ausdrücklich für die aufwändige wissenschaftliche Arbeit des Autors Prof. Dr. Lutz Thieme. Im Ergebnis beschreibt der Autor eine relevante Übersterblichkeit erfolgreicher Sportlerinnen und Sportler aus Deutschland, am deutlichsten ausgeprägt bei Olympiasiegern und in neuerer Zeit.
Der WR erkennt die besondere Wichtigkeit und Relevanz des vorgelegten Ansatzes, der dabei helfen kann, potenzielle Gesundheitsrisiken bei Hochleistungssportarten zu identifizieren. Aus einer medizinischen Perspektive wäre es jedoch zusätzlich zur rein beschreibenden Statistik notwendig, Einblick in die jeweiligen Todesursachen zu erhalten. Erst dann könnte versucht werden, Ursache und Wirkung zu analysieren, um auf dieser Grundlage eventuelle Konsequenzen zum Schutz der Athleten und Athletinnen zu benennen. Eine dieser Konsequenzen sollte sein, das eng gewobene medizinische Betreuungssystem im Leistungssport auch über das Karriereende hinaus zu institutionalisieren. Präventiv-sportmedizinische Untersuchungen werden im Kadersport des DOSB und der Landessportverbände nur für aktive Sportlerinnen und Sportler organisiert. Nach dem Ende der Karriere werden durch die Sportorganisationen mit ganz wenigen Ausnahmen kei- ne leistungssportspezifischen Präventionsangebote mehr vermittelt.

Ein besonderer und fraglos lebensverkürzender Aspekt ergibt sich aus der Anwendung von Dopingpraktiken, deren Identifikation jedoch im präventiven Bereich offensichtliche Schwierigkeiten entgegenstehen. Das Dopingopfer-Hilfegesetz greift ,nur “ bei Fällen von Zwangsdoping und beinhaltet weniger präventiv-medizinische Hilfen als vielmehr eine finanzielle Kompensation erlittener Gesundheitsschäden. Es bleibt auch angesichts der vorgelegten Mortalitätsstatistik unklar, in welchem Ausmaß Folgeschäden von Doping für eine eventuelle Übersterblichkeit im Sport tatsächlich verantwortlich sind.

Die vorgelegte Studie steht - wie vom Autor selbst benannt - zudem im Widerspruch zur umfänglichen internationalen Literatur. So suggeriert die Arbeit einen negativen Einfluss der deutschen Olympiateilnahme auf die spätere Lebenserwartung - die bisherige internationale Studienlage zeigt jedoch, dass 
Leistungssportler eine generell erhöhte Lebenserwartung aufweisen. Als wahrscheinlichste Gründe hierfür werden eine lebenslang erhöhte körperliche Aktivität, ein gesünderer Lebensstil oder die genetische Selektion analysiert und diskutiert (Kontro, Sarna, Kaprio, \& Kujala, 2018). Selbst die Teilnehmer der Tour de France (hinsichtlich Risikobereitschaft oder Dopinganwendung vermutlich mit einem erhöhten Gesundheitsrisiko behaftet) zeigten in der retrospektiven Bewertung keinerlei Übersterblichkeit (Marijon et al., 2013). Auch weitere hochrangig publizierte und begutachtete wissenschaftliche Studien haben bisher keine entsprechende Übersterblichkeit bei (Ex-)Athleten und Athletinnen gezeigt (Teramoto \& Bungum, 2010; Garatachea et al., 2014; Antero-Jacquemin et al., 2015; Kontro et al., 2018; Takeuchi et al., 2019). Vielmehr wird durchgehend von einer erhöhten Lebenserwartung berichtet, so auch in der großen Arbeit von Clarke et al. (2015) mit Untersuchungsdaten von 15.174 Medaillengewinnern/ -innen der Olympischen Spiele - übrigens hier auch speziell in der Subgruppe deutscher Olympioniken. Allenfalls aus dem nichtolympischen Wettkampfsport gibt es Berichte, wie z. B. vom Powerlifting (Pärssinen, Kujala, Vartiainen, Sarna, \& Seppälä, 2000), das bei finnischen Athleten mit einer eindeutig erhöhten Mortalität assoziiert war; in diesem Fall erklärt durch eine besonders hohe Inzidenz von Anabolikamissbrauch in dieser extremen Kraftsportart.

Entweder liegt dem Studienergebnis also tatsächlich eine bisher nicht erkannte Besonderheit deutscher Sportlerinnen und Sportler oder des deutschen Sportsystems zugrunde, oder die Ergebnisse müssen aufgrund einiger methodischer Unsicherheiten deutlich vorsichtiger interpretiert werden, als dies der Autor der Studie selbst getan hat.

Einige dieser methodischen Aspekte möchten wir beispielhaft herausgreifen:

- Die untersuchten Teilgruppen der vorgelegten Studie sind zum Teil sehr klein. So werden durch einzelne Fälle (die ja zufällig auftreten können) sehr große Änderungen in der Statistik provoziert. Je erfolgreicher, desto kleiner werden die Teilgruppen in der Studie - was die beobachtete Zunahme der Mortalität mit steigendem sportlichem Erfolg auch ohne Gesundheitsprobleme erklären kann. Häufig fehlen auch konkrete Angaben zu statistischen Fehlern ( $p$ Werte, Konfidenzintervalle). Es ist daher kaum möglich, zu entscheiden, ob Unterschiede allein aufgrund zufälliger Schwankungen zustande kamen oder ob es sich um reale Effekte handelt.

- Da Sterbedaten naturgemäß nur von verstorbenen Personen vorliegen, die verbleibende Lebensdauer noch lebender Personen aber offenbleibt, entsteht eine gewisse statistische Unsicherheit. Die Gesamtmortalität kann mit letzter Sicherheit erst beschrieben werden, wenn eine Gruppe von Personen insgesamt verstorben ist. In der Studie wurden durchaus spezielle Methoden verwendet, um diese Unsicherheit zu kompensieren und die Mortalität gleichsam anhand der vorliegenden Stichprobe zu schätzen. Dennoch wird die Unsicherheit einer solchen Schätzung natürlich umso größer, je jünger das betrachtete Kollektiv war - weil dann weniger Personen tatsächlich verstorben sind. So ist z. B. die in Tab. 1 verwendete Lebensdauer Verstorbener kein geeignetes Maß für Vergleiche der Überlebenszeit der gesamten Kollektive (da die noch Lebenden dabei ignoriert werden).

- Der Abhängigkeit von Einflussfaktoren widmet der Autor keine ausreichende Aufmerksamkeit. So wurde der Vergleich BRD gegen DDR nicht für eine eventuelle Beeinflussung durch die in Tab. 3 identifizierten und als relevant benannten Faktoren korrigiert. Auch diese Vergleiche sind daher nur mit allergrößter Vorsicht $\mathrm{zu}$ interpretieren.

Trotz dieser methodischen Einschränkungen erkennt der WR die Wichtigkeit der Arbeit und die hohe Relevanz des Themenfelds. Aufgrund der komplexen Datenlage (die allermeisten der betreffenden Sportler leben ja noch) müssten nach unserer Ansicht die Ergebnisse jedoch deutlich zurückhaltender interpre- tiert und diskutiert werden, als dies in der Publikation selbst geschehen ist. Bis die Aussage möglich ist, dass Hochleistungssport bei deutschen Sportlern tatsächlich zu einer späteren Übersterblichkeit führt, wäre es notwendig, Ursachen, Wirkungen und Kausalketten zu prüfen, um daraus Handlungsempfehlungen für ein gezieltes, das sportmedizinisch-präventive Betreuungssystem des DOSB auf die Zeit nach der Karriere erweiternde System abzuleiten. Dies sollte aus unserer Sicht ausdrücklich eine weitere Intensivierung von Dopingprävention einschließen.

Eine konkrete und generelle Lebenszeitverkürzung für alle deutschen Teilnehmer am Spitzensport kann der WR auch angesichts der publizierten Daten nicht erkennen. Nichtsdestotrotz halten wir weitere Analysen, insbesondere unter Einbeziehung der Todesursache, für folgerichtig.

\section{Der Wissenschaftsrat der DGSP (in alphabetischer Reihenfolge)}

Prof. Dr. Wilhelm Bloch, Köln Prof. Dr. Anja Hirschmüller, Rheinfelden Prof. Dr. Frank Mayer, Potsdam Prof. Dr. Andreas Nieß, Tübingen Prof. Dr. Tim Meyer, Saarbrücken Prof. Dr. Dr. Claus Reinsberger, Paderborn

Prof. Dr. Kai Röcker, Furtwangen Prof. Dr. Jürgen M. Steinacker, Ulm

Wir danken für die biometrische Beratung durch Prof. Dr. Dr. Ulrich Abel.

\section{Korrespondenzadresse

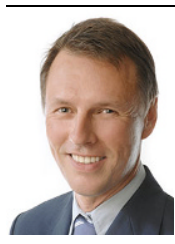 \\ Prof. Dr. Kai Röcker Institut für Angewandte Gesundheitsförderung und Bewegungsmedizin, Hochschule Furtwangen Robert-Gerwig-Platz 1, 78120 Furtwangen, Deutschland kai.roecker@hs-furtwan- gen.de}

Funding. Open Access funding enabled and organized by Projekt DEAL.

Interessenkonflikt. W. Bloch, A. Hirschmüller F. Mayer, A. Nieß, T. Meyer, C. Reinsberger, K. Röcker 
und J.M. Steinacker geben an, dass kein Interessenkonflikt besteht.

Open Access. Dieser Artikel wird unter der Creative Commons Namensnennung 4.0 International Lizenz veröffentlicht, welche die Nutzung, Vervielfältigung, Bearbeitung, Verbreitung und Wiedergabe in jeglichem Medium und Format erlaubt, sofern Sie den/die ursprünglichen Autor(en) und die Quelle ordnungsgemäß nennen, einen Link zur Creative Commons Lizenz beifügen und angeben, ob Änderungen vorgenommen wurden.

Die in diesem Artikel enthaltenen Bilder und sonstiges Drittmaterial unterliegen ebenfalls der genannten Creative Commons Lizenz, sofern sich aus der Abbildungslegende nichts anderes ergibt. Sofern das betreffende Material nicht unter der genannten Creative Commons Lizenz steht und die betreffende Handlung nicht nach gesetzlichen Vorschriften erlaubt ist, ist für die oben aufgeführten Weiterverwendungen des Materials die Einwilligung des jeweiligen Rechteinhabers einzuholen.

Weitere Details zur Lizenz entnehmen Sie bitte der Lizenzinformation auf http://creativecommons.org/ licenses/by/4.0/deed.de.

\section{Literatur}

Antero-Jacquemin, J., Rey, G., Marc, A., et al. (2015). Mortality in female and male French Olympians: a 1948-2013 cohort study. Am J Sports Med, 43(6), 1505-1512. https://doi.org/10.1177/ 0363546515574691.

Clarke, P.M., Walter, S.J., Hayen, A., Mallon, W. J., Heijmans, J., \& Studdert, D. M. (2015). Survival of the fittest: retrospective cohort study of the longevity of Olympic medallists in the modern era. Br J Sports Med, 49(13), 898-902. https://doi. org/10.1136/bjsports-2015-e8308rep.

Garatachea, N., Santos-Lozano, A., Sanchis-Gomar, F., et al. (2014). Elite athletes live longer than the general population: a meta-analysis. Mayo Clin Proc,89(9), 1195-1200. https://doi.org/10.1016/ j.mayocp.2014.06.004

Kontro, T. K., Sarna, S., Kaprio, J., \& Kujala, U. M. (2018). Mortality and health-related habits in 900 Finnish former elite athletes and their brothers. Br J Sports Med, 52(2), 89-95. https://doi.org/10. 1136/bjsports-2017-098206.

Marijon, E., Tafflet, M., Antero-Jacquemin, J., El Helou, N., Berthelot, G., Celermajer, D. S., et al. (2013). Mortality of French participants in the Tour de France (1947-2012). European Heart Journal, 34(40), 3145-3150. https://doi.org/10.1093/ eurheartj/eht347.

Pärssinen, M., Kujala, U., Vartiainen, E., Sarna, S., \& Seppälä, T. (2000). Increased premature mortality of competitive powerlifters suspected to have used anabolic agents. Int J Sports Med, 21(3), 225-227. https://doi.org/10.1055/s2000-304.

Takeuchi, T., Kitamura, Y., Sado, J., et al. (2019). Mortality of Japanese Olympic athletes: 1952-2017 cohort study. BMJ Open Sport Exerc Med, 5(1), e653. https://doi.org/10.1136/bmjsem-2019-000653.

Teramoto, M., \& Bungum, T. J. (2010). Mortality and longevity of elite athletes. J Sci Med Sport, 13(4), 410-416. https://doi.org/10.1016/j.jsams.2009. 04.010 . 